\title{
A previously uncharacterized gene, yjfO (bsmA), influences Escherichia coli biofilm formation and stress response
}

\begin{abstract}
Correspondence
Robert J. C. McLean

McLean@txstate.edu
\end{abstract}

Received 4 June 2009

Revised 5 October 2009

Accepted 14 October 2009

\author{
Mary M. Weber, ${ }^{1} \dagger$ Christa L. French, ${ }^{1} \ddagger$ Mary B. Barnes, ${ }^{2}$ \\ Deborah A. Siegele ${ }^{3}$ and Robert J. C. McLean ${ }^{1}$
}

\author{
${ }^{1}$ Department of Biology, Texas State University-San Marcos, 601 University Drive, San Marcos, \\ TX 78666, USA \\ ${ }^{2}$ Tulane National Primate Research Center, 18703 Three Rivers Road, Covington, LA 70433-8915, \\ USA \\ ${ }^{3}$ Department of Biology, Texas A\&M University, College Station, TX 77843-3258, USA
}

\begin{abstract}
Bacteria growing as surface-adherent biofilms are better able to withstand chemical and physical stresses than their unattached, planktonic counterparts. Using transcriptional profiling and quantitative PCR, we observed a previously uncharacterized gene, yjfO to be upregulated during Escherichia coli MG1655 biofilm growth in a chemostat on serine-limited defined medium. A yjfO mutant, developed through targeted-insertion mutagenesis, and a yjfO-complemented strain, were obtained for further characterization. While bacterial surface colonization levels (c.f.u. $\mathrm{cm}^{-2}$ ) were similar in all three strains, the mutant strain exhibited reduced microcolony formation when observed in flow cells, and greatly enhanced flagellar motility on soft $(0.3 \%)$ agar.

Complementation of yjfO restored microcolony formation and flagellar motility to wild-type levels. Cell surface hydrophobicity and twitching motility were unaffected by the presence or absence of $y j f O$. In contrast to the parent strain, biofilms from the mutant strain were less able to resist acid and peroxide stresses. yjfO had no significant effect on $E$. coli biofilm susceptibility to alkali or heat stress. Planktonic cultures from all three strains showed similar responses to these stresses.

Regardless of the presence of $y j f O$, planktonic $E$. coli withstood alkali stress better than biofilm populations. Complementation of yjfO restored viability following exposure to peroxide stress, but did not restore acid resistance. Based on its influence on biofilm maturation and stress response, and effects on motility, we propose renaming the uncharacterized gene, yjfO, as bsmA (biofilm stress and motility).
\end{abstract}

\section{INTRODUCTION}

When growing as surface-adherent, biofilm communities, bacteria are typically quite resistant to a variety of adverse environmental conditions, including antimicrobial agents, $\mathrm{pH}$ extremes and oxidative stresses (reviewed by Costerton et al., 1987). Biofilms have been implicated in a number of problems, including many infections, industrial fouling, and corrosion (Costerton et al., 1987; McLean et al., 1996).

tPresent address: Department of Microbial and Molecular Pathogenesis, Texas A\&M Health Sciences Center, College Station, TX 77840-7896, USA.

$\ddagger$ Present address: Applied Biosystems/Ambion, 2130 Woodward St, Austin, TX 78744-1832, USA.

Abbreviation: TEM, transmission electron microscopy.

The gene array data discussed in this paper have been deposited in Gene Expression Omnibus (GEO) under accession number GSE18362.
Several detailed studies have been conducted on biofilms to ascertain the differences between planktonic and biofilm bacteria. In Gram-negative bacteria, these studies include large-scale mutant screens using microtitre (O'Toole \& Kolter, 1998b; Prigent-Combaret et al., 1999) and competition culture assays (Junker et al., 2006), promoter identification using in vivo expression technology (Finelli et al., 2003), proteomic analyses (Sauer et al., 2002), and transcription profiling (Domka et al., 2007; Hancock \& Klemm, 2007; Junker et al., 2007; Ren et al., 2004; Schembri et al., 2003; Whiteley et al., 2001a). The use of these techniques has allowed the identification of genes important for biofilms, such as those associated with cell signalling involving acylated homoserine lactones (Herzberg et al., 2006; Lee et al., 2007a), osmotic stress and reduced oxygen (Prigent-Combaret et al., 1999), and the global regulators rpoS (Adams \& McLean, 1999), relA and spoT (Balzer \& McLean, 2002). 
Many of the genes expressed at higher levels in biofilms than in planktonic cells encode proteins of unknown function. A number of these are now being associated with various biofilm phenotypes. In Pseudomonas aeruginosa, $n d v B$ is responsible for the tobramycin resistance of biofilms (Mah et al., 2003). In Escherichia coli, ariR $(y m g B)$ is needed for biofilm formation and for full expression of acid resistance genes in biofilms (Lee et al., 2007b). The E. coli bhsA $(y c f R)$ gene is needed for stimulation of biofilm formation by stresses such as hydrogen peroxide, low $\mathrm{pH}$ and heat stress (Zhang et al., 2007). In the present study, we observed the uncharacterized gene $y j f O$ (b4189) to be upregulated. This same gene has been found to be upregulated in at least two previous transcriptional profiling studies (Beloin et al., 2004; Junker et al., 2007). Here, we show that yjfO mutants are altered in biofilm structure and cell motility, and in their ability as biofilms to respond to $\mathrm{pH}$ and oxidative stresses.

\section{METHODS}

Bacterial strains and media. The strains and plasmids used in this study are listed in Table 1. The insertion mutation in strain B4189 was confirmed upon receipt by PCR. Cultures were maintained on LuriaBertani (LB) agar (MG1655), LB supplemented with $50 \mu \mathrm{g}$ kanamycin $\mathrm{ml}^{-1}$ (mutant strain), or LB supplemented with $50 \mu \mathrm{g}$ kanamycin $\mathrm{ml}^{-1}$ and $100 \mu \mathrm{g}$ ampicillin $\mathrm{ml}^{-1}$ (complemented strain). For long-term preservation, overnight cultures were frozen at $-80{ }^{\circ} \mathrm{C}$ using glycerol [final concentration $12.5 \%(\mathrm{v} / \mathrm{v})$ ] as a cryoprotectant. Prior to experimentation, cultures were revived from frozen stock, cultured overnight on LB agar, and then transferred to MOPS serine medium (Neidhardt et al., 1974). This minimal medium, used in other biofilm studies (Junker et al., 2007; Schembri et al., 2003), contained serine $\left(1 \mathrm{mg} \mathrm{ml}^{-1}\right)$ as the carbon source. Additional amino acids (Ile, Arg, Gly, His, Leu, Met, Phe, Val and Thr, each at $40 \mu \mathrm{g}$ $\mathrm{ml}^{-1}$ ) were also present. All cultures were grown at $37^{\circ} \mathrm{C}$, using laboratory facilities at Texas State University.

Chemostat culture. The biofilm culture chemostat apparatus has been previously described (Whiteley et al., 1997). Briefly, it consists of a chemostat from which the culture can be circulated through a biofilm culture device (in this case $4 \mathrm{~m}$ Tygon laboratory tubing or a flow cell). For culturing, the chemostat was filled with MOPS serine medium, as described above (Neidhardt et al., 1974), inoculated with $1 \mathrm{ml}$ of an overnight culture of E. coli and grown at $37{ }^{\circ} \mathrm{C}$ as a batch culture for $24 \mathrm{~h}$, after which continuous culture was initiated with a peristaltic pump at a dilution rate of $0.025 \mathrm{~h}^{-1}$. The culture was allowed to equilibrate for one full generation $(40 \mathrm{~h})$. At this point, biofilm growth was initiated as a second pump continuously circulated the chemostat culture through the $4 \mathrm{~m}$ length of attached tubing $\left(100 \mathrm{ml} \mathrm{h}^{-1}\right)$ for $96 \mathrm{~h}$. Bacteria attached to the tubing were considered to be the biofilm culture, whereas unattached cells represented the planktonic culture.

Cell harvesting. For planktonic cell harvesting, $200 \mathrm{ml}$ culture was mixed with an equal volume of ice-cold $\left(-20{ }^{\circ} \mathrm{C}\right)$ stop solution $[5 \%$ $(\mathrm{v} / \mathrm{v})$ water-saturated phenol in ethanol] to stop endogenous nuclease activity, and then placed into nuclease-free $50 \mathrm{ml}$ centrifuge tubes (Falcon) (Arnold et al., 2001). Cells were harvested by centrifugation $\left(3200 \mathrm{~g}, 4{ }^{\circ} \mathrm{C}\right.$ for $\left.20 \mathrm{~min}\right)$, frozen $\left(-80{ }^{\circ} \mathrm{C}\right)$, and then transported in liquid nitrogen to Texas A\&M University for RNA extraction and analysis.

For biofilm cell harvesting, the biofilm-colonized tubing was removed from the chemostat, drained to remove planktonic cells, and then filled with ice-cold stop solution. Following this, the tubing was cut into $2 \mathrm{~cm}$ sections, each section was cut in half and placed into a sterile Petri plate, and biofilm cultures were scraped from the tubing with a sterile scalpel into $200 \mathrm{ml}$ ice-cold stop solution. The scraped tubing sections were then placed into $200 \mathrm{ml}$ ice-cold stop solution and sonicated in a bath sonicator (Sonicor Instrument Corporation) at $60 \mathrm{~Hz}$ for $10 \mathrm{~min}$ to further dislodge biofilm cells. Biofilm cells were then harvested from the stop solution by centrifugation, frozen, and transported to Texas A\&M University for RNA extraction and analysis as described above.

Biofilm growth measurements. In order to measure biofilm and planktonic cell growth, duplicate chemostat cultures were established for each strain as described above. Following growth, the culture was analysed by dilution plating to enumerate the planktonic populations. For biofilm growth, the biofilm-colonized tubing was removed and rinsed with sterile medium, to remove loosely attached cells. The tubing was cut into five $2 \mathrm{~cm}$ sections, placed into sterile PBS, sonicated at $60 \mathrm{~Hz}$ for $5 \mathrm{~min}$, and vortexed for $2 \mathrm{~min}$ to dislodge biofilm cells (McLean et al., 1999). Cell numbers were then measured by dilution plating onto LB agar.

RNA processing and gene array analysis. RNA extraction and purification were conducted at Texas A\&M University using a previously described hot phenol extraction protocol (Arnold et al., 2001) and enzymic purification. $\left[{ }^{33} \mathrm{P}\right] \mathrm{CTP}$ (New England Biochemical) was used to label cDNA during reverse transcription (Arnold et al., 2001). Gene array analysis was conducted using the Sigma Genosys macroarray protocol previously described by Arnold et al. (2001). For differential expression patterns to be considered significant, a minimum twofold change in expression level (compared with the background) during both the original and replicate run was needed.

Real-time (quantitative) PCR. Transcriptional profiling results for yjfO were validated using real-time PCR (Ju et al., 2007). RNA was purified as described above, and reverse-transcribed to generate cDNA using a high-capacity cDNA archive kit (Applied Biosystems) using the manufacturer's protocol. The primers used for quantitative PCR were: TM-B4189-204F (5'-ACC GCC AGT AAC GGA CCA T$\left.3^{\prime}\right)$ and TM-B4189-313R (5' -CTA ATG CGT CAT CCG GAG AAC$\left.3^{\prime}\right)$, and $5^{\prime}-/ 5(6)$-FAM/CCA TCG TGC TTA CGC TAC CTA TTC GCT GTA/36-TAMTph/-3' (Integrated DNA Technologies) was used as

Table 1. E. coli strains and plasmids used in this study

\begin{tabular}{|lll|}
\hline \multicolumn{1}{|c|}{ Characteristics } & \multicolumn{1}{c|}{ Source and reference } \\
\hline MG1655 (DS291) & Wild-type $\left(\mathrm{F}^{-} \lambda^{-}\right.$rph-1) & M. Cashel, NIH (Hernandez \& Cashel, 1995) \\
B4189 (FB22940) & MG1655 yjfO:: Tn5(KAN-I-SceI) at position & F. R. Blattner, University of Wisconsin (Kang et al., 2004) \\
& 167 in minus orientation & This study \\
pMW201 & pGEM-T containing $y j f O$ & \\
\hline
\end{tabular}


a probe [5(6)-FAM $=5^{\prime} 6$-carboxyfluorescein; 36TAMpH=3'TAMRA]. No template and no reverse transcriptase controls were used. cDNA products were measured using an Applied Biosystems Prism 7500 realtime PCR system. Real-time analysis was conducted in duplicate.

Epifluorescence microscopy. To compare biofilm formation of the various strains, each strain was grown in a chemostat coupled to a three-chamber flow cell (Stovall Life Science). The chemostat was inoculated and equilibrated as described above. For biofilm growth, the culture was pumped through the flow cell at $100 \mathrm{ml} \mathrm{h}^{-1}$. After $96 \mathrm{~h}$ biofilm growth, the flow cell was removed from the chemostat and each chamber was washed with $5 \mathrm{ml}$ sterile water to remove planktonic cells. Cells were stained with $20 \mu \mathrm{M}$ Syto 9 (Invitrogen) for $30 \mathrm{~min}$, and then rinsed with $5 \mathrm{ml}$ sterile water. Biofilms were viewed using a Nikon Eclipse 80 I microscope. Images were obtained using a Nikon DXM 1200F digital camera and images were analysed using Image-Pro Plus (version 5.1) (Mirza et al., 2007). Adobe Photoshop CS3 (version 10.0.1) was used to convert the images to greyscale, invert the image (to make bacteria appear dark against a light background), and optimize contrast. Each flow cell experiment was carried out in triplicate.

Transmission electron microscopy (TEM). In order to observe whether the presence or absence of $y j f O$ affected cell surface stability or flagella production, cells were examined by negative-stain preparations with TEM. Overnight cultures were grown on agar plates and then a loop-full of culture was suspended in a water droplet on Parafilm. The cell suspension was then mixed with $1 \%(\mathrm{w} / \mathrm{v})$ uranyl acetate, placed on a Formvar-coated grid, and examined by TEM (Merchant et al., 2007). TEM films were scanned (2700 dots per inch) using a photographic high-resolution scanner, and the images were inverted and optimized for contrast using Adobe Photoshop CS3 as described above.

Twitching and flagellar motility assays. In order to elicit differences in twitching motility between the strains, the methods outlined by Semmler et al. (1999) were employed. Each strain was stabbed into the centre of an LB agar plate containing $1 \%(\mathrm{w} / \mathrm{v})$ agar and incubated at $37{ }^{\circ} \mathrm{C}$ in an inverted position. To examine flagellabased motility, cultures were stab-inoculated into the centre of an LB soft agar $(0.3 \%$, w/v, agar) plate (O'Toole \& Kolter, 1998a) and the plates incubated in an upright position at $37{ }^{\circ} \mathrm{C}$. Colony diameter due to twitching motility and flagella motility was measured after $16 \mathrm{~h}$ incubation.

Hydrophobicity assay. Strains were tested for their ability to partition into hexane from an aqueous suspension as described by Zhang et al. (2007).

Biofilm stress assays. We evaluated the bacterial responses to $\mathrm{pH}$, hydrogen peroxide and elevated temperature stresses using the experimental strategy of Zhang et al. (2007). For this purpose, each strain was again cultured in a chemostat, coupled to laboratory tubing as described above. In order to evaluate biofilm stress responses, the biofilm-colonized tubing was removed and cut into $2 \mathrm{~cm}$ pieces. Sensitivity to acidic and alkaline conditions was assessed by placing five pieces of tubing into $\mathrm{pH} 2.5$ or $\mathrm{pH} 12$ MOPS serine medium, which was subsequently incubated at $37{ }^{\circ} \mathrm{C}$ for $20 \mathrm{~min}$. To determine viability following exposure to hydrogen peroxide, five pieces of tubing were incubated for $5 \mathrm{~min}$ at $37{ }^{\circ} \mathrm{C}$ in $20 \mathrm{mM} \mathrm{H}_{2} \mathrm{O}_{2}$. To assess viability following exposure to heat, five pieces of tubing were placed into MOPS serine medium and incubated for $10 \mathrm{~min}$ at $65{ }^{\circ} \mathrm{C}$. Following incubation, the tubing was placed into PBS, which was subsequently sonicated at $60 \mathrm{~Hz}$ for $5 \mathrm{~min}$ and vortexed for $2 \mathrm{~min}$. Each sample was serially diluted and plated on LB. A total of three biological replicates were performed for each stress measurement.
Planktonic stress assays. In order to determine strain viability following exposure to numerous environmental stressors, the methods outlined by Zhang et al. (2007) were employed. All strains were incubated in MOPS serine medium at $37{ }^{\circ} \mathrm{C}$ with shaking at 100 r.p.m. to $\mathrm{OD}_{600} 0.3$. To assess viability at altered $\mathrm{pH}, 2 \mathrm{ml}$ culture was incubated for an additional hour at $37{ }^{\circ} \mathrm{C}$ without shaking in $\mathrm{pH} 2.5$ or $\mathrm{pH} 12$ MOPS serine medium. To determine culture viability following exposure to hydrogen peroxide, $1 \mathrm{ml}$ culture was incubated with $20 \mathrm{mM} \mathrm{H} \mathrm{O}_{2}$ at $37{ }^{\circ} \mathrm{C}$ without shaking for $15 \mathrm{~min}$. For heat sensitivity, $5 \mathrm{ml}$ of each strain was removed and heated for $20 \mathrm{~min}$ at $65{ }^{\circ} \mathrm{C}$. Following each treatment, cultures were serially diluted and plated on $\mathrm{LB}$, and incubated at $37{ }^{\circ} \mathrm{C}$ for $24 \mathrm{~h}$. A minimum of three biological replicates were performed.

Data analysis. On the basis of dilution plating, the planktonic and biofilm cell concentrations were calculated as c.f.u. $\mathrm{ml}^{-1}$ and c.f.u. $\mathrm{cm}^{-2}$, respectively. For statistical analyses, the data were logtransformed (Whiteley et al., 2001b) and the data analysed by oneway ANOVA, with a minimum threshold of significance of $P<0.05$. Where applicable, all pair-wise comparisons were analysed by the Holm-Sidak method using SigmaStat v3.0 (Systat Software). Several measurements gave no detectable survival and in these cases, the c.f.u. data for that particular sample were assigned a value of 1 c.f.u. (the $\log _{10}$ of an undetectable sample was therefore zero). A minimum of three biological replicates was performed for each measurement. SigmaPlot v8.0 (Systat) was used to plot the results.

Complementation of $\boldsymbol{E}$. coli mutant. The $y j f O$ gene was amplified from MG1655 using the primers yjfO-F1 (5'-GATGTGGGTTACGCTTTCGT-3'), yjfO-R1 (5'-CCACTGTCCTGTCACGATG$3^{\prime}$ ) using AmpliTaq Gold (Applied Biosystems). Purified yjfO gene products were cloned into the pGEM-T Easy Vector (Promega) to generate pMW201, where $y j f O$ could be transcribed from the lac promoter through induction with $0.1 \mathrm{mM}$ IPTG. The resulting plasmid was subsequently electroporated into the corresponding mutant strain. Verification of the inserted gene was accomplished through restriction digestion using EcoRI and plasmid sequencing. IPTG was present in the MOPS serine medium during chemostat experiments with the complemented strain.

\section{RESULTS}

\section{Gene array}

In order to investigate differences in biofilm and planktonic culture gene expression, we conducted transcriptional profiling on $96 \mathrm{~h}$ biofilms of E. coli. We found that 43 genes were differentially expressed between biofilm and planktonic culture. In the literature, the number of genes that are differentially expressed within biofilms in comparison with planktonic cultures ranges from a low of approximately $0.5 \%$ of the genome (Whiteley et al., 2001a) to a high of almost $20 \%$ (Hancock \& Klemm, 2007; Ren et al., 2004). The values obtained in the present study (43 genes) (GenBank accession number GSE18362) represent approximately $1 \%$ of the genome (Blattner et al., 1997) and are certainly consistent with other reports. As noted elsewhere (Beloin et al., 2004; Ren et al., 2004; Schembri et al., 2003), many of the differentially regulated genes were uncharacterized. Several initially uncharacterized genes, identified in earlier transcriptional profiling investigations, have since been shown to be important in biofilm 
functions (Domka et al., 2007; Zhang et al., 2007). Under our experimental conditions, expression of yjfO (b4189) was $3.3 \pm 0.3$-fold higher in biofilms than in planktonic culture, as measured by transcriptional profiling. Upregulation of $y j f O$ in biofilms was confirmed by quantitative PCR [ $\Delta$ threshold cycle $\left.\left(\mathrm{C}_{\mathrm{t}}\right) 5.0 \pm 0.5\right]$. Two previous studies have also shown $y j f O$ to be upregulated in biofilms (Beloin et al., 2004; Junker et al., 2007).

\section{Biofilm formation}

To investigate the role of $y j f O$ in biofilm formation, we circulated a steady-state $E$. coli culture through a threechambered flow cell for $96 \mathrm{~h}$. The flow cell was removed after $96 \mathrm{~h}$, stained with $20 \mu \mathrm{M}$ Syto 9 and viewed using a Nikon Eclipse $80 \mathrm{I}$ microscope at $\times 100$ magnification. Clumping and microcolony formation were observed in the wild-type (MG1655) biofilms (Fig. 1a). This phenotype was absent in the $y j f O$ mutant (Fig. 1b), but could be restored upon genetic complementation (Fig. 1c). In order to measure adherent cell populations, we grew each of the strains in a chemostat coupled to $4 \mathrm{~m}$ Tygon laboratory tubing for biofilm culture (Whiteley et al., 1997). Following $96 \mathrm{~h}$ of biofilm growth, the tubing was removed and assayed for colonization by dilution plating as described above. No statistically significant difference was noted in planktonic or adherent cell populations among any of the strains (Fig. 2). Based on these observations we concluded that $y j f O$ expression is important in microcolony formation and biofilm maturation processes, but not necessary for planktonic growth or initial surface adhesion (Beloin et al., 2004; Sauer et al., 2002).

\section{Motility and ultrastructure}

As twitching motility has been shown to be important for microcolony formation (O'Toole \& Kolter, 1998a), we investigated whether the phenotype of the $y j f O$ deletion could be explained by loss of this characteristic. Although there were slight differences in colony expansion (Fig. 3a), these differences were not significant when analysed by one-way ANOVA $(P=0.63)$, indicating that $y j f O$ had no effect on twitching motility. In contrast, flagella-based motility was enhanced in the $y j f O$ mutant and the complemented but not induced strain $(P<0.001$ compared with the wild type), but could be restored to wild-type levels when the $y j f O$-complemented strains were induced by IPTG (Fig. 3b). Using TEM, we examined negatively stained preparations of the various strains for the presence of flagella and found an indication of enhanced flagella in some of the $y j f O$ mutants (Fig. 4). In future studies, we will explore the mechanism(s) for this yjfO-enhanced flagellabased motility.

Based on its sequence, the YjfO protein is predicted to be a lipoprotein (Rudd et al., 1998), and as such has the potential to be associated with cell membranes. Cell preparation during negative-stain TEM involves suspend-
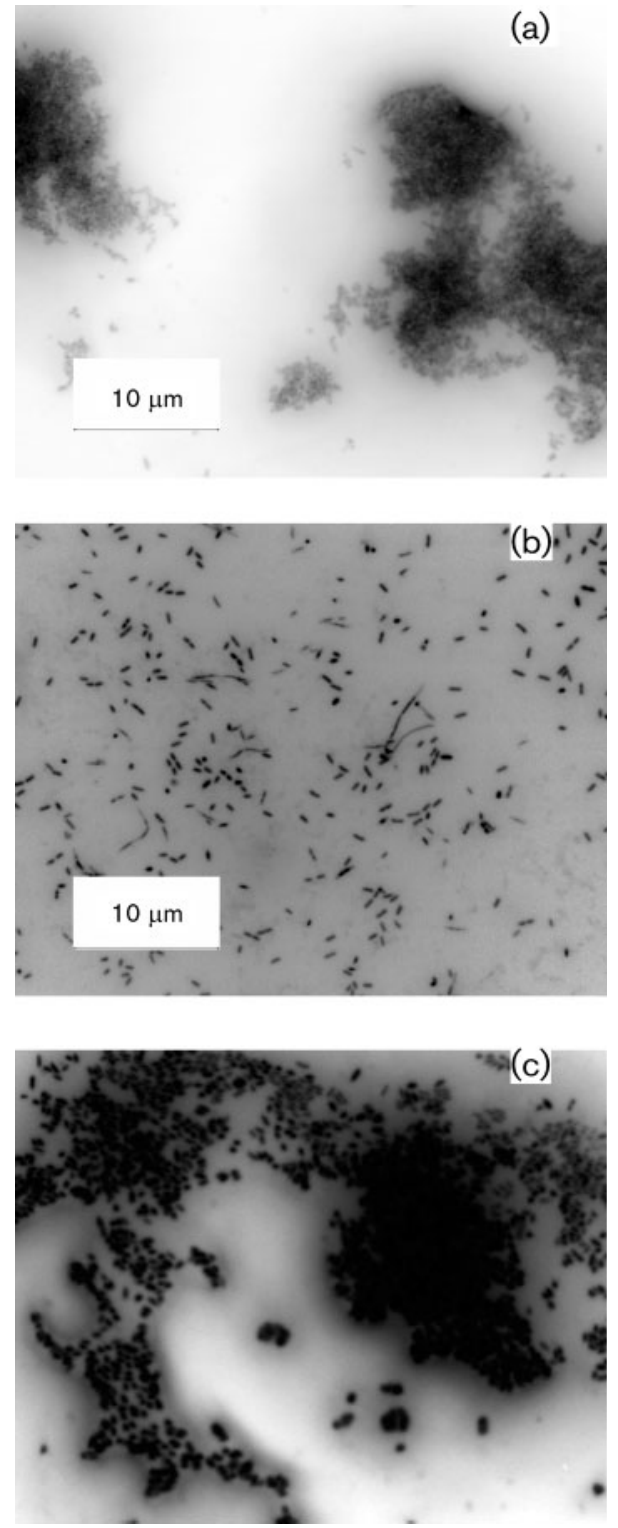

Fig. 1. Representative epifluorescence microscopy images of $96 \mathrm{~h}$ biofilms from chemostat cultures grown in serine-limited MOPS medium: MG1655 (a), yjfO (b4189) (b) and complemented $y j f O(c)$. The magnification is the same in all figures.

ing the bacteria in a heavy metal solution (to provide electron contrast), and then exposing the stained, unfixed cells to high vacuum during TEM examination. Other EM preparation approaches, such as conventional embedding or freeze substitution (Graham \& Beveridge, 1990), employ chemical fixation and gradual dehydration, processes which stabilize and protect membranes and other structures from the high vacuum and electron beam in TEM. As fixation and gentle dehydration protocols are not used during routine negative-stain TEM preparation, one would anticipate that membranes lacking a key structural component would be much more prone to vacuum- 


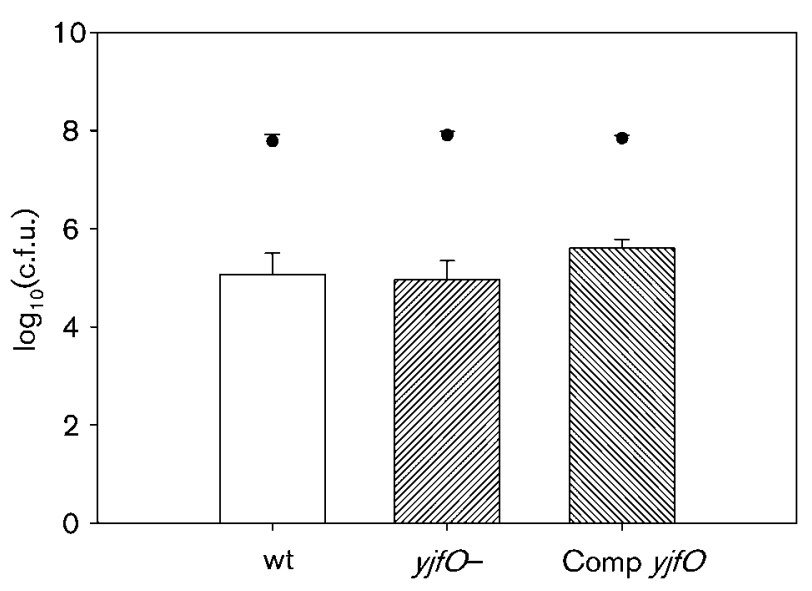

Fig. 2. Planktonic (scatter plot) and biofilm growth (bar graph) of MG1655, yjfO (b4189) (yjfO-) and complemented yjfO (Comp yjfO) after $96 \mathrm{~h}$ chemostat growth. Values in all figures are expressed as $\log _{10}$ (c.f.u. $\mathrm{ml}^{-1}$ ) for planktonic cultures and $\log _{10}$ (c.f.u. $\mathrm{cm}^{-2}$ ) for biofilm cultures ( \pm SEM). No significant differences were seen among the planktonic populations or biofilm populations of the three strains.
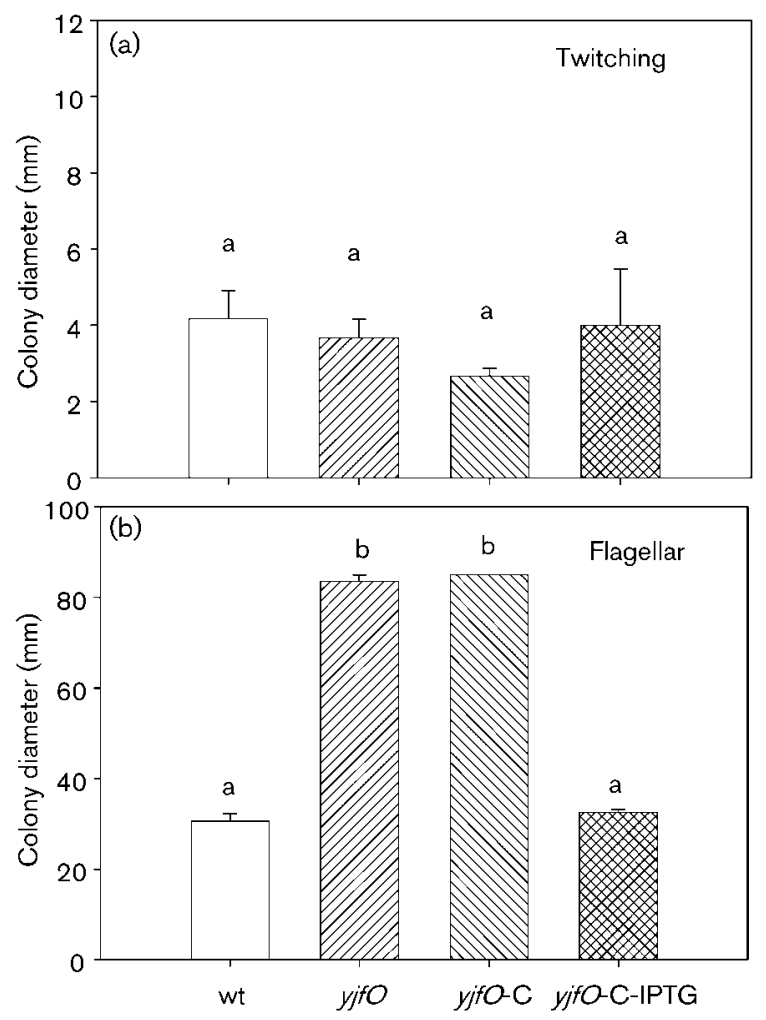

Fig. 3. Twitching (a) and flagella-based (b) motility in MG1655

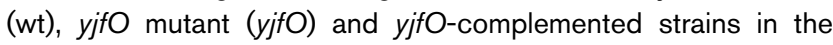
absence (yjfO-C) and presence (yjfO-C-IPTG) of IPTG induction. Values with the same letter, in all figures, are not significantly different $(P<0.05)$. induced damage. However, we did not observe any major differences in the cell envelope membranes, regardless of the presence (Fig. 4a, c) or absence of yjfO (Fig. 4b). However, we cannot rule out subtle, membrane-associated changes due to $y j f O$ solely on the basis of negative-stain TEM examination.

\section{Cell surface hydrophobicity}

In order to assess the effect of $y j f O$ on cell surface hydrophobicity, aqueous suspensions of the E. coli strains were mixed with an equal volume of hexane, and the fraction of cells remaining in the aqueous layer was measured as described elsewhere (Zhang et al., 2007). Following this procedure, the percentage of cells $( \pm \mathrm{SE})$ remaining in the aqueous phase was: wild-type $86.5(0.5)$, yjfO $81.5(0.5)$, complemented $y j f O$ without IPTG 82.5 (0.5), and complemented and IPTG-induced yjfO 92 (3). Based on these results, yjfO appears to have had a limited effect on cell surface hydrophobicity in these experiments. However, recent work by Q. Ma and T. K. Wood (unpublished results, personal communication) has shown that $y j f O$ overexpression in another E. coli strain results in greatly increased hydrophobicity. As a result, we cannot completely rule out a contribution of $y j f O$ to cell surface hydrophobicity.

\section{Planktonic and biofilm stress assays}

In order to determine the viability of each strain following exposure to commonly encountered environmental stresses, chemostat-grown biofilm populations were exposed to acid, base, hydrogen peroxide (oxidative stress) or heat stress. As shown in Fig. 2, no statistically significant differences in overall cell numbers were noted between the strains in the absence of stress, thus allowing for standardization and efficient determination of biofilm cell viability following exposure to each stressor.

Exposure of $96 \mathrm{~h}$ biofilms to $\mathrm{pH} 2.5$ MOPS serine medium (acid stress) resulted in a decrease in overall viability for all strains assayed. In the planktonic populations (Fig. 5a), the acid-treated wild-type cells were reduced in number in comparison with the untreated control; however, the difference was only marginally significant $(P=0.099)$. The other two populations (yjfO mutant and complemented strain) were significantly reduced in comparison with the untreated control, with the $y j f O$ strain showing the biggest reduction and the complemented strain showing a sensitivity intermediate between those of the wild-type and the $y j f O$ mutant. However, the acid-treated planktonic strains did not differ significantly with respect to each other. In contrast to the planktonic results, the viability patterns in the biofilm cultures following acid stress were quite different (Fig. 6a), with all populations differing significantly from each other. In comparison with the acidtreated wild-type biofilms, yjfO mutant viability was reduced approximately 200 -fold. The viability of the 

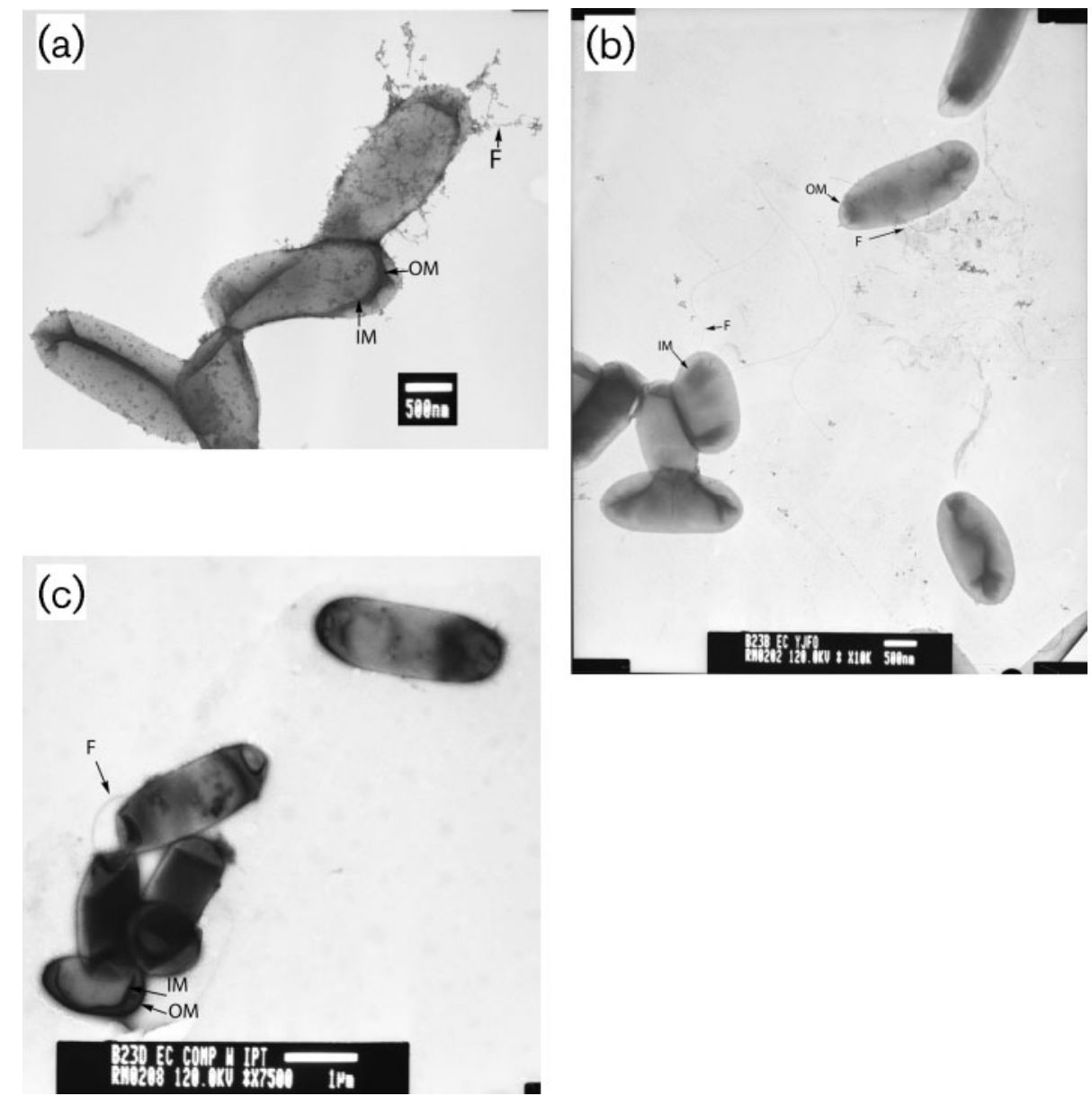

Fig. 4. TEM micrographs of negative-stain preparations of $E$. coli MG1655 (a), yjfO (b), and yjfO-complemented and IPTGinduced strains (c). Flagella (F), outer (OM) and inner (IM) membranes are indicated. Bars: $0.5 \mu \mathrm{m}(\mathrm{a}, \mathrm{b}), 1.0 \mu \mathrm{m}(\mathrm{c})$.

complemented strain was reduced even further (approximately sixfold in comparison with the mutant). The failure of pMW201 to complement the acid-sensitive phenotype of the mutant suggests that the $y j f O$ insertion mutation has polar effects on expression of the adjacent downstream gene $y j f N$. Given that the complemented strain survives less well than the mutant, it is also possible that high levels of YjfO increase sensitivity to low $\mathrm{pH}$.

Exposure of 96 h biofilms to pH 12 MOPS serine medium (base stress) resulted in decreased viability of $E$. coli biofilms (Fig. 6b). Here, all three strains (wild-type, mutant and complemented strain) showed a statistically similar reduction in viability. In contrast, there was no significant stress-induced change in viability in planktonic cultures (Fig. 5b). The increased susceptibility of biofilm populations to an environmental stress (in this case, alkaline $\mathrm{pH}$ ), in comparison with planktonic populations, is highly unusual in that the converse is generally the case (Costerton et al., 1987; Davey \& O'Toole, 2000). We will investigate this issue in future studies.
Exposure of planktonic and biofilm populations to oxidative stress $\left(20 \mathrm{mM} \mathrm{H}_{2} \mathrm{O}_{2}\right)$ resulted in a statistically significant decrease in viability only in the $y j f O$ mutant biofilm, which was complemented by the reintroduction of the $y i f O$ gene (Fig. $6 \mathrm{c}$ ). In planktonic cultures, there was a modest, and statistically insignificant, reduction in the viability of the $y j f O$ mutant (Fig. 5c). We interpret these results as demonstrating that $y j f O$ is involved in the protection of biofilms against oxidative stress.

In contrast to the above-mentioned stresses, we observed no significant differences in planktonic (Fig. 5d) or biofilm (Fig. 6d) culture viability following exposure to heat $\left(65{ }^{\circ} \mathrm{C}\right)$.

\section{DISCUSSION}

E. coli is routinely found in soil, water and intestinal mucus, all of which impose unique stresses on the organism in both biofilm and planktonic modes of growth (Fabich et al., 2008). In intestinal mucus, E. coli encounters 

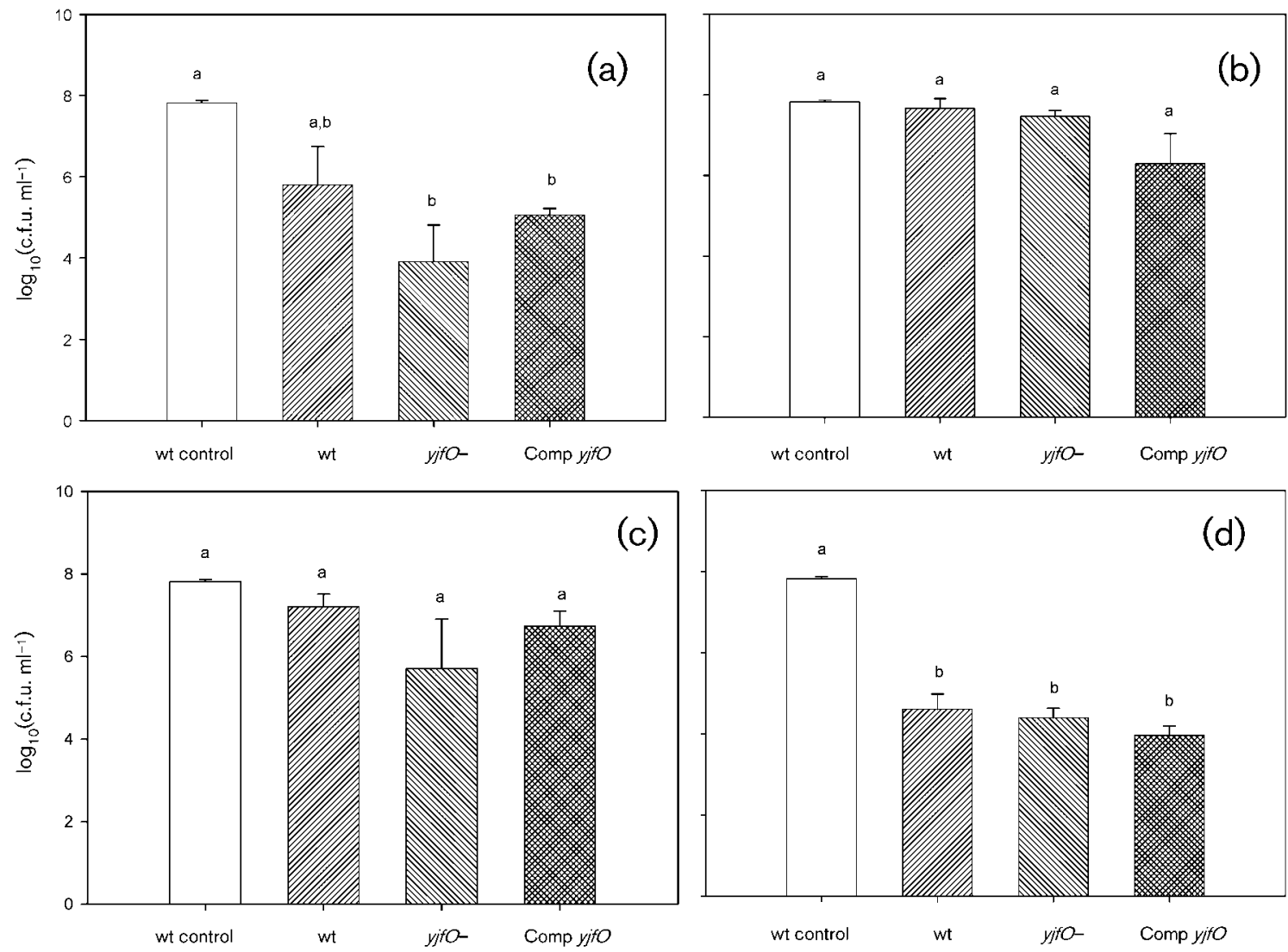

Fig. 5. Survival of E. coli planktonic cells following exposure to acid (a), base (b), oxidative stress (c) and heat stress (d). Strains in this figure are designated untreated wild-type control (wt control), treated MG1655 (wt), yjfO (b4189) (yjfO-), and complemented and induced yjfO (Comp yjfO). The value for the untreated control corresponds to the average of the wild-type planktonic cells without exposure to any stressor and is included for viability comparisons.

acetate and other volatile fatty acids, produced as metabolic by-products of the resident flora (Arnold et al., 2001). These organic acids are capable of traversing the membrane into the bacterial cell, thus inducing acid stress (Arnold et al., 2001). Typically, these weak acids decrease bacterial viability, yet strains of E. coli, including MG1655 (Arnold et al., 2001), are capable of combating them. Acid resistance is one factor that allows $E$. coli to efficiently colonize and inhabit the intestinal tract. In this same largely anaerobic environment, E. coli would be exposed to transient concentrations of reactive oxygen species from mucosal innate defences (McLean et al., 1988). In contrast, E. coli does not experience alkaline conditions (above $\mathrm{pH} 8$ ) in its normal environment, and so the low resistance to alkaline stress is anticipated. The surprising observation in the current study was the increased resistance of planktonic populations (Fig. 5b) to alkali stress when compared with biofilm populations (Fig. 6b). Also, heat susceptibility was unaffected by biofilm (Fig. 6d) or planktonic growth (Fig. 5d). Although biofilm growth is normally associated with stress resistance (Costerton et al.,
1987; Davey \& O'Toole, 2000), our current study certainly shows exceptions to this phenomenon.

In this study, we focused on $y j f O$, a member of the $y h c N$ family. When first described (Rudd et al., 1998), the $y h c N$ family consisted of nine parahomologous, uncharacterized genes $(y j f O, y a h O, y b i J, y b i M, y c f R, y d g H, y h c N, y j f N$ and $y j f Y$ ) of unknown function. Members of this family are predicted to have evolved from a common ancestor, based on the presence of a signal peptide and a shared motif in their N and C termini (Rudd et al., 1998). One of these genes, $y c f R$, has been shown to be upregulated in biofilm cultures and to be associated with biofilm stress responses (Zhang et al., 2007). On that basis, Zhang et al. (2007) have proposed that this gene be renamed to $b h s A$ for influencing biofilms through hydrophobicity and stress response. In the present study, $y j f O$ does contribute to the biofilm stress response but unlike $y c f R$ does not appear to contribute to cell surface hydrophobicity.

Cell aggregation and microcolony formation of surfaceadherent bacteria via twitching motility and other 

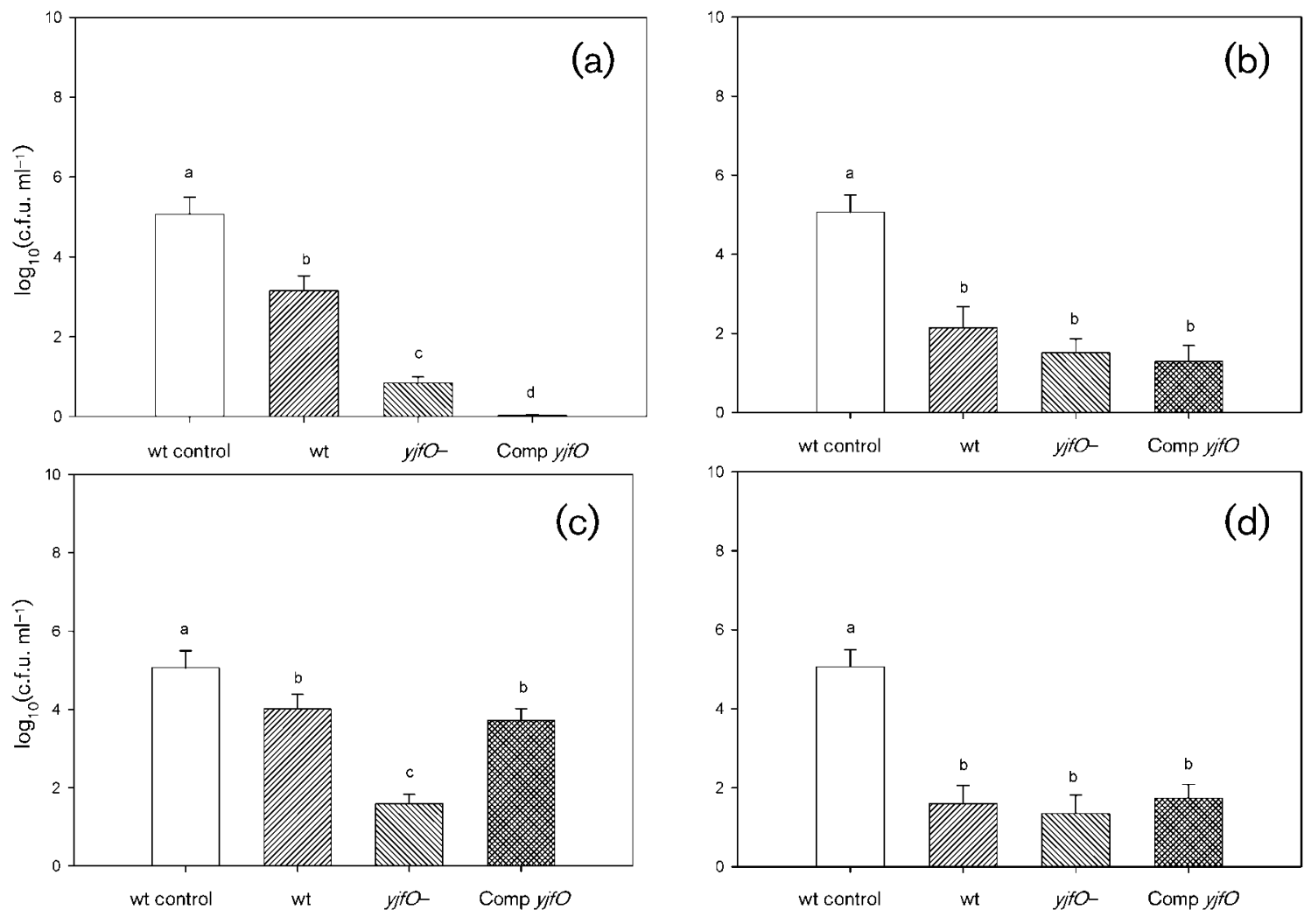

Fig. 6. Survival of E. coli biofilm cells following exposure to acid stress (a), base stress (b), oxidative stress (c) and heat stress (d). For strain designations, see legend to Fig. 5. The value for the untreated control corresponds to the average of the wild-type biofilm cells without exposure to any stressor and is included for viability comparisons.

processes are hallmarks of early biofilm maturation (O’Toole \& Kolter, 1998a; Sauer et al., 2002). In the current study (Fig. 1), we noted that wild-type E. coli formed microcolonies within flow cells. This feature was absent in the $y j f O$ mutant biofilm, although it was restored upon genetic complementation. However, adherent cell concentrations were similar (Fig. 2). Aside from twitching and flagella motility (O’Toole \& Kolter, 1998a), other characteristics involved in cell aggregation include cell-cell adhesion, cell signalling (Domka et al., 2006) and hydrophobic interactions (McEldowney \& Fletcher, 1986). The most striking feature of $y j f O$ deletion was the loss of microcolony formation (Fig. 1) and greatly enhanced flagella motility (Fig. 3). Other investigators have shown the importance of microcolonies, water channels and other biofilm structures in the resistance of the component organisms to various stresses (Matz et al., 2004; Pamp \& Tolker-Nielsen, 2007). Although flagella motility has been shown to be important in biofilm structure development (Wood et al., 2006), our present study suggests that unchecked flagella motility disrupts microcolonies, a feature that certainly provides a plausible explanation for the greatly reduced biofilm stress response. Due to its contribution to biofilm stress response as well as its contribution to flagella motility, we propose renaming yjfO as bsmA (biofilm stress and motility).

\section{ACKNOWLEDGEMENTS}

This work was funded by grants from the National Institutes of Health (1R15 AI050638) to R. J.C.M. and (RO1 GM55154) to D.A.S., and from the Texas Higher Education Coordinating Board Advanced Research Program (003615-0037-2007) to R. J. C. M. We thank Kerry Fuson, Qun Ma and Patricia Zenker for assistance, and Jean Marc Ghigo, Anthony Hay, Karl Klose, Ron Walter, Marvin Whiteley and Tom Wood for helpful discussions and advice. R. J. C. M. would like to dedicate this paper to the memory of Blanche A. V. McLean.

\section{REFERENCES}

Adams, J. L. \& McLean, R. J. C. (1999). The impact of rpoS deletion on Escherichia coli biofilms. Appl Environ Microbiol 65, 4285-4287.

Arnold, C. N., McElhanon, J., Lee, A., Leonhart, R. \& Siegele, D. A. (2001). Global analysis of Escherichia coli gene expression during the acetate-induced acid tolerance response. J Bacteriol 183, 2178-2186.

Balzer, G. J. \& McLean, R. J. C. (2002). The stringent response genes relA and spoT are important for Escherichia coli biofilms under slowgrowth conditions. Can J Microbiol 48, 675-680.

Beloin, C., Valle, J., Latour-Lambert, P., Faure, P., Kzreminski, M., Balestrino, D., Haagensen, J. A., Molin, S., Prensier, G. \& other authors (2004). Global impact of mature biofilm lifestyle on Escherichia coli K-12 gene expression. Mol Microbiol 51, 659-674.

Blattner, F. R., Plunkett, G., III, Bloch, C. A., Perna, N. T., Burland, V., Riley, M., Collado-Vides, J., Glasner, J. D., Rode, C. K. \& other 
authors (1997). The complete genome sequence of Escherichia coli K12. Science 277, 1453-1474.

Costerton, J. W., Cheng, K.-J., Geesey, G. G., Ladd, T. I., Nickel, J. C., Dasgupta, M. \& Marrie, T. J. (1987). Bacterial biofilms in nature and disease. Annu Rev Microbiol 41, 435-464.

Davey, M. E. \& O'Toole, G. A. (2000). Microbial biofilms: from ecology to molecular genetics. Microbiol Mol Biol Rev 64, 847-867.

Domka, J., Lee, J. \& Wood, T. K. (2006). YliH (BssR) and YceP (BssS) regulate Escherichia coli K-12 biofilm formation by influencing cell signaling. Appl Environ Microbiol 72, 2449-2459.

Domka, J., Lee, J., Bansal, T. \& Wood, T. K. (2007). Temporal geneexpression in Escherichia coli K-12 biofilms. Environ Microbiol 9, 332-346.

Fabich, A. J., Jones, S. A., Chowdhury, F. Z., Cernosek, A., Anderson, A., Smalley, D., McHargue, J. W., Hightower, G. A., Smith, J. T. \& other authors (2008). Comparison of carbon nutrition for pathogenic and commensal Escherichia coli strains in the mouse intestine. Infect Immun 76, 1143-1152.

Finelli, A., Gallant, C. V., Jarvi, K. \& Burrows, L. L. (2003). Use of inbiofilm expression technology to identify genes involved in Pseudomonas aeruginosa biofilm development. J Bacteriol 185, 2700-2710.

Graham, L. L. \& Beveridge, T. J. (1990). Evaluation of freezesubstitution and conventional embedding protocols for routine electron microscopic processing of eubacteria. J Bacteriol 172, 21412149.

Hancock, V. \& Klemm, P. (2007). Global gene expression profiling of asymptomatic bacteriuria Escherichia coli during biofilm growth in human urine. Infect Immun 75, 966-976.

Hernandez, V. J. \& Cashel, M. (1995). Changes in conserved region 3 of Escherichia coli $\sigma^{70}$ mediate ppGpp-dependent functions in vivo. J Mol Biol 252, 536-549.

Herzberg, M., Kaye, I. K., Peti, W. \& Wood, T. K. (2006). YdgG (TqsA) controls biofilm formation in Escherichia coli K-12 through autoinducer 2 transport. J Bacteriol 188, 587-598.

Ju, Z., Wells, M. C., Heater, S. J. \& Walter, R. B. (2007). Multiple tissue gene expression analyses in Japanese medaka (Oryzias latipes) exposed to hypoxia. Comp Biochem Physiol C Toxicol Pharmacol 145, 134-144.

Junker, L. M., Peters, J. E. \& Hay, A. G. (2006). Global analysis of candidate genes important for fitness in a competitive biofilm using DNA-array-based transposon mapping. Microbiology 152, 2233-2245.

Junker, L. M., Toba, F. A. \& Hay, A. G. (2007). Transcription in Escherichia coli PHL628 biofilms. FEMS Microbiol Lett 268, 237-243.

Kang, Y., Durfee, T., Glasner, J. D., Qiu, Y., Frisch, D., Winterberg, K. M. \& Blattner, F. R. (2004). Systematic mutagenesis of the Escherichia coli genome. J Bacteriol 186, 4921-4930.

Lee, J., Jayaraman, A. \& Wood, T. K. (2007a). Indole is an interspecies biofilm signal mediated by SdiA. BMC Microbiol 7, 42.

Lee, J., Page, R., García-Contreras, R., Palermino, J. M., Zhang, X. S., Doshi, O., Wood, T. K. \& Peti, W. (2007b). Structure and function of the Escherichia coli protein YmgB: a protein critical for biofilm formation and acid-resistance. J Mol Biol 373, 11-26.

Mah, T. F., Pitts, B., Pellock, B., Walker, G. C., Stewart, P. S. \& O'Toole, G. A. (2003). A genetic basis for Pseudomonas aeruginosa biofilm antibiotic resistance. Nature 426, 306-310.

Matz, C., Bergfeld, T., Rice, S. A. \& Kjelleberg, S. (2004). Microcolonies, quorum sensing and cytotoxicity determine the survival of Pseudomonas aeruginosa biofilms exposed to protozoan grazing. Environ Microbiol 6, 218-226.

McEldowney, S. \& Fletcher, M. (1986). Variability of the influence of physicochemical factors affecting bacterial adhesion to polystyrene substrata. Appl Environ Microbiol 52, 460-465.
McLean, R. J. C., Nickel, J. C., Cheng, K.-J. \& Costerton, J. W. (1988). The ecology and pathogenicity of urease-producing bacteria in the urinary tract. Crit Rev Microbiol 16, 37-79.

McLean, R. J. C., Fortin, D. \& Brown, D. A. (1996). Microbial metal binding mechanisms and their relation to nuclear waste disposal. Can J Microbiol 42, 392-400.

McLean, R. J. C., Whiteley, M., Hoskins, B. C., Majors, P. D. \& Sharma, M. M. (1999). Laboratory techniques for studying biofilm growth, physiology, and gene expression in flowing systems and porous media. Methods Enzymol 310, 248-264.

Merchant, M. M., Welsh, A. K. \& McLean, R. J. C. (2007). Rheinheimera texasensis sp. nov., a halointolerant freshwater oligotroph. Int J Syst Evol Microbiol 57, 2376-2380.

Mirza, B. S., Welsh, A. \& Hahn, D. (2007). Saprophytic growth of inoculated Frankia sp. in soil microcosms. FEMS Microbiol Ecol 62, 280-289.

Neidhardt, F. C., Bloch, P. L. \& Smith, D. F. (1974). Culture medium for Enterobacteria. J Bacteriol 119, 736-747.

O'Toole, G. A. \& Kolter, R. (1998a). Flagellar and twitching motility are necessary for Pseudomonas aeruginosa biofilm development. Mol Microbiol 30, 295-304.

O'Toole, G. A. \& Kolter, R. (1998b). Initiation of biofilm formation in Pseudomonas fluorescens WCS365 proceeds via multiple, convergent signaling pathways: a genetic analysis. Mol Microbiol 28, 449-461.

Pamp, S. J. \& Tolker-Nielsen, T. (2007). Multiple roles of biosurfactants in structural biofilm development by Pseudomonas aeruginosa. J Bacteriol 189, 2531-2539.

Prigent-Combaret, C., Vidal, O., Dorel, C. \& Lejeune, P. (1999). Abiotic surface sensing and biofilm-dependent regulation of gene expression in Escherichia coli. J Bacteriol 181, 5993-6002.

Ren, D., Bedzyk, L. A., Thomas, S. M., Ye, R. W. \& Wood, T. K. (2004). Gene expression in Escherichia coli biofilms. Appl Microbiol Biotechnol 64, 515-524.

Rudd, K. E., Humphrey-Smith, I., Wasinger, V. C. \& Bairoch, A. (1998). Low molecular weight proteins: a challenge for post-genomic research. Electrophoresis 19, 536-544.

Sauer, K., Camper, A. K., Ehrlich, G. D., Costerton, J. W. \& Davies, D. G. (2002). Pseudomonas aeruginosa displays multiple phenotypes during development as a biofilm. J Bacteriol 184, 1140-1154.

Schembri, M. A., Kjærgaard, K. \& Klemm, P. (2003). Global gene expression in Escherichia coli biofilms. Mol Microbiol 48, 253-267.

Semmler, A. B. T., Whitchurch, C. B. \& Mattick, J. S. (1999). A reexamination of twitching motility in Pseudomonas aeruginosa. Microbiology 145, 2863-2873.

Whiteley, M., Brown, E. \& McLean, R. J. C. (1997). An inexpensive chemostat apparatus for the study of microbial biofilms. J Microbiol Methods 30, 125-132.

Whiteley, M., Bangera, M. G., Bumgarner, R. E., Parsek, M. R., Teitzel, G. M., Lory, S. \& Greenberg, E. P. (2001a). Gene expression in Pseudomonas aeruginosa biofilms. Nature 413, 860-864.

Whiteley, M., Ott, J. R., Weaver, E. A. \& McLean, R. J. C. (2001b). Effects of community composition and growth rate on aquifer biofilm bacteria and their susceptibility to betadine disinfection. Environ Microbiol 3, 43-52.

Wood, T. K., Gonzalez Barrios, A. F., Herzberg, M. \& Lee, J. (2006). Motility influences biofilm architecture in Escherichia coli. Appl Microbiol Biotechnol 72, 361-367.

Zhang, X. S., Garcia-Contreras, R. \& Wood, T. K. (2007). YcfR (BhsA) influences Escherichia coli biofilm formation through stress response and surface hydrophobicity. J Bacteriol 189, 3051-3062.

Edited by: V. Sperandio 